\title{
Patient reported outcomes for non-tuberculous mycobacterial disease
}

\author{
Michael R. Loebinger ${ }^{1,2}$ and Surinder S. Birring ${ }^{3}$ \\ Affiliations: ${ }^{1}$ Royal Brompton and Harefield NHS Foundation Trust, London, UK. ${ }^{2}$ National Heart and Lung \\ Institute, Imperial College, London, UK. ${ }^{3}$ Centre for Human and Applied Physiological Sciences, School of \\ Basic and Medical Biosciences, Faculty of Life Sciences and Medicine, King's College London, London, UK.
}

Correspondence: Michael R. Loebinger, Royal Brompton Hospital, Host Defence, Sydney St, London, SW3 6NP, UK. E-mail: m.loebinger@rbht.nhs.uk

@ERSpublications

There is a pressing need for patient related outcome measures for pulmonary NTM disease, to assist with both clinical management decisions and as an endpoint for clinical studies. This NTM Module should be considered an important step in this development. http://bit.ly/2OvmAQw

Cite this article as: Loebinger MR, Birring SS. Patient reported outcomes for non-tuberculous mycobacterial disease. Eur Respir J 2020; 55: 1902204 [https://doi.org/10.1183/13993003.02204-2019].

The morbidity and mortality from non-tuberculous mycobacterial (NTM) disease is increasing [1]. Treatment of NTM usually requires multidrug regimens and is often associated with poor tolerability, significant side-effects and high failure rates. Not all patients with pulmonary NTM disease need treatment, and deciding who and when to treat can be challenging. The management of NTM is significantly hampered by the lack of reliable and responsive biomarkers to assess disease activity, progression and response to therapy, and at present, clinical decisions are made with a combination of symptom, radiology and microbiological assessments. There is a pressing need for new therapies and approaches for pulmonary NTM disease, and this will require robust clinical endpoints to evaluate them.

The ultimate aim of NTM treatment is to eradicate the organism, leading to a cure. Therefore, a microbiological endpoint, such as sustained culture conversion, is extremely important and must be the gold standard, as with other diseases such as TB. This was the endpoint used in the large, multicentre, randomised controlled trial in pulmonary NTM, that led to the only licensed drug for this condition [2]. There are, however, limitations to this endpoint. For clinical management, the accuracy of culture results is dependent on the quality of the sputum, and results take up to 8 weeks, making management changes dependent on prior clinical state. Additionally, successful conversion may not necessarily lead to a clinical and symptomatic improvement. The significant number of untreated NTM patients that remain well highlights this discordance [3]. The US Food and Drug Administration acknowledges this and has indicated that new treatments should improve the way a patient feels, functions or survives [4]. Furthermore, there is a significant relapse and reinfection rate in pulmonary NTM disease, which again may impact on the value of culture conversion, if it is not sustained [5]. It is also important to note that a cure or culture conversion may not be the aim in some patients' treatment, whereby stability or symptomatic improvement may be the primary goal.

Patient related outcome (PRO) measures that assess symptoms and health-related quality of life (HRQOL) are important tools as they assess the patients' perspective and complement objective tools. They can be used in clinical management to determine disease severity, progression or response, and may predict prognosis and have the potential to select patients for treatment [6]. PROs may also provide responsive clinical trial endpoints to be able to formally assess the impact of therapies. There is presently no 
symptom or HRQOL PRO for pulmonary NTM disease. A range of HRQOL PROs have been evaluated in NTM, such as the St Georges' Respiratory Questionnaire (SGRQ), COPD Assessment Test (CAT), and Short Form 36 Health Survey Questionnaire (SF-36) [7-9]. The limitation of such tools is that they are not NTM disease-specific and were developed for use in COPD and general health. The consequence of using generic tools is that they may not be as responsive to change compared to disease-specific tools and often contain redundant items, increasing the questionnaire administration time.

The timely article by HeNkLe et al. [10] in this issue of the European Respiratory Journal describes the preliminary validation of a patient reported outcome measure assessing non-pulmonary symptoms in patients with pulmonary NTM. The NTM Module items were selected following patient and physician focus groups and open-ended interviews with patients. The module consists of 20 items arranged into four domains (NTM Symptoms, Body Image, Digestive Symptoms and Eating Problems). 203 patients from a single centre with pulmonary Mycobacterium avium complex (MAC) disease and no cystic fibrosis, that had met the American Thoracic Society/Infectious Disease Society of America (ATS/IDSA) pulmonary NTM disease criteria [11] at any point were included, which meant that some patients had been previously treated and were now culture negative. The NTM module had good internal consistency and reproducibility over 2 weeks. It was able to discriminate patients according to disease severity defined by the presence of cavitation or active disease. A longitudinal analysis was performed in 76 of the patients, with the module repeated on two occasions, a year apart. Three of the four domains of NTM module were responsive in the 35 patients that started treatment. Although it would have been helpful to note how these scores varied depending on other clinical outcome measures, it is likely that the patients selected for the second PRO measure at 1 year were those that had remained on treatment and were hence doing well clinically and tolerating the medication. The NTM module is therefore a very welcome development since it is a disease-specific tool and it has some encouraging clinical characteristics required to perform well in clinical trials.

There are, however, some concerns too that suggest the NTM Module may require further refinement for optimisation. The development of the NTM Module has not been peer-reviewed and published and it is not clear how items were selected in the final NTM Module, and if psychometric methodology, such as item response theory and Rasch analysis, were used to assist this to achieve an instrument with linear scaling properties [12].

It is a PRO developed for the digestive and systemic symptoms of NTM disease, including 20 items which focused on smell, taste, sleep, weather effects, and abdominal and weight symptoms and issues. It is possible that some of these items relate to the side-effects of particular medications, rather than the underlying disease process. Surprisingly, this tool does not capture respiratory symptoms, which are necessary to fulfil the ATS/IDSA and British Thoracic Society criteria for pulmonary disease, and the main symptomatic burden of this disease, with cough, sputum, dyspnoea and haemoptysis, in addition to lethargy and malaise commonly reported [11, 13]. The authors recommend using the described NTM Module alongside another PRO; QOL-B, which was designed for patients with bronchiectasis, or the CFQ-R for cystic fibrosis $[14,15]$.

It is possible that the NTM Module may work well alongside the QOL-B and CFQ-R, but there are several important concerns that limit this assumption. Neither of these tools were designed specifically for NTM disease. The current study did not evaluate the patient's perspective of the combined tool and it is therefore not known if important concepts were missed, or if there is significant redundancy of items (likely, as data shows particularly with CFQ-R). The current study should have validated the NTM and QOL-B from the start, rather than mid-study, and therefore only a few subjects were assessed in a subgroup analysis. Importantly, the psychometric properties of the combined tool have not been reported, such as ceiling and floor effects, internal consistency, repeatability and so on. The combined NTM and QOL-B tool now comprises 12 domains and more than 50 items, which is quite long for an instrument and it is very likely that a single tool for NTM disease, designed from scratch, would have been briefer and potentially be inclusive of pulmonary symptoms. Additionally, patients with NTM may have neither cystic fibrosis nor bronchiectasis, making both suggested additional tools inappropriate.

The NTM Module could be used as a stand-alone evaluation of digestive and systemic symptoms. There are some limitations for this too. The NTM Module has high ceiling effects, affecting two of the four domains: eating problems and body image (a total of seven items). The consequence of this is that one of the domains is clearly not responsive to change, as the data suggests. Obvious problems with this are that they would be unable to show positive responses to treatment. Furthermore, the module does not have a total health status score, and this may present difficulties when evaluating the overall efficacy of treatment in clinical trials.

Despite these concerns, this article is an important advance in an area desperate for more management options. There is a pressing need for a PRO measure for pulmonary NTM disease, to assist with both 
clinical management decisions and as an endpoint for clinical studies. The study by HenkLe et al. [10] demonstrates the first development of an NTM tool with good reproducibility and responsiveness, and should be considered an important step towards developing a validated PRO for pulmonary NTM.

Conflict of interest: M.R. Loebinger reports personal fees from Insmed, Savara, Grifols, Bayer, Raptor, Polyphor and AstraZeneca, outside the submitted work. S.S. Birring has nothing to disclose.

\section{References}

1 Cowman S, van Ingen J, Griffith DE, et al. Non-tuberculous mycobacterial pulmonary disease. Eur Respir J 2019; 54: 1900250 .

2 Olivier KN, Griffith DE, Eagle G, et al. Randomized trial of liposomal amikacin for inhalation in nontuberculous mycobacterial lung disease. Am J Respir Crit Care Med 2017; 195: 814-823.

3 Hwang JA, Kim S, Jo KW, et al. Natural history of Mycobacterium avium complex lung disease in untreated patients with stable course. Eur Respir J 2017; 49: 1600537.

4 US Department of Health and Human Services, Food and Drug Administration. Guidance for Industry: Patient-Reported Outcome Measures: Use in medical Product Development to Support Labeling Claims. Silver Spring, FDA, 2009.

5 Wallace RJ Jr, Brown-Elliott BA, McNulty S, et al. Macrolide/azalide therapy for nodular/bronchiectatic Mycobacterium avium complex lung disease. Chest 2014; 146: 276-282.

6 Hunter AM, Campbell IA, Jenkins PA, et al. Treatment of pulmonary infections caused by mycobacteria of the Mycobacterium avium-intracellulare complex. Thorax 1981; 36: 326-329.

7 Hama M, Ushiki A, Kosaka M, et al. Health-related quality of life in patients with pulmonary non-tuberculous mycobacteria infection. Int J Tuberc Lung Dis 2016; 20: 747-752.

8 Czaja CA, Levin AR, Cox CW, et al. Improvement in quality of life after therapy for Mycobacterium abscessus group lung infection. A prospective cohort study. Ann Am Thorac Soc 2016; 13: 40-48.

9 Mehta M, Marras TK. Impaired health-related quality of life in pulmonary nontuberculous mycobacterial disease. Respir Med 2011; 105: 1718-1725.

10 Henkle E, Winthrop KL, Ranches GP, et al. Preliminary validation of the NTM Module: a patient-reported outcome measure for patients with pulmonary nontuberculous mycobacterial disease. Eur Respir J 2020; 55: 1901300.

11 Griffith DE, Aksamit T, Brown-Elliott BA, et al. An official ATS/IDSA statement: diagnosis, treatment, and prevention of nontuberculous mycobacterial diseases. Am J Respir Crit Care Med 2007; 175: 367-416.

12 Tennant A, McKenna SP, Hagell P. Application of Rasch analysis in the development and application of quality of life instruments. Value Health 2004; 7: Suppl. 1, S22-S26.

13 Haworth CS, Banks J, Capstick T, et al. British Thoracic Society guidelines for the management of non-tuberculous mycobacterial pulmonary disease (NTM-PD). Thorax 2017; 72: iil-ii64.

14 Quittner AL, Buu A, Messer MA, et al. Development and validation of The Cystic Fibrosis Questionnaire in the United States: a health-related quality-of-life measure for cystic fibrosis. Chest 2005; 128: 2347-2354.

15 Quittner AL, O'Donnell AE, Salathe MA, et al. Quality of Life Questionnaire-Bronchiectasis: final psychometric analyses and determination of minimal important difference scores. Thorax 2015; 70: 12-20. 